\title{
Comunicación
}

\section{Eucoleosis por Eucoleus boehmi en un canino con elevadas cargas parasitarias}

\section{Eucoleus boehmi infection in a dog with high parasitic burdens}

\author{
Fernando Adrián Fariña ${ }^{1,2,3}$, Mariana Inés Pasqualetti ${ }^{1,2}$, \\ Mariano Emmanuel Ercole ${ }^{1,2}$,Clara Bessi ${ }^{1,2}$, Mabel Ribicich ${ }^{1,2}$
}

\section{Resumen}

Eucoleus boehmi (sin. Capillaria boehmi) es un parásito nematodo trichurideo del tracto respiratorio superior en cánidos y de curso generalmente asintomático. Se presenta el caso clínico de un canino macho mestizo de 9 años de edad, procedente de la provincia de Buenos Aires, Argentina, que había sido tratado previamente por eucoleosis, que presentaba tos, estornudos, estornudos inversos, abundante secreción nasal serosa bilateral. Se realizó un análisis de materia fecal por las técnicas de Bembrook y McMaster revelando la presencia de $10900 \mathrm{hpg}$ de E. boehmi. Se realizó el tratamiento y seguimiento del paciente hasta la remisión completa de los signos clínicos y la ausencia de huevos en materia fecal.

Palabras clave: Eucoleus boehmi, caninos, HPG, eucoleosis

\footnotetext{
${ }^{1}$ Universidad de Buenos Aires, Facultad de Ciencias Veterinarias, Cátedra de Parasitología y Enfermedades Parasitarias, Buenos Aires, Argentina

${ }^{2}$ CONICET - Universidad de Buenos Aires, Facultad de Ciencias Veterinarias, Instituto de Investigaciones en Producción Animal (INPA), Buenos Aires, Argentina

${ }^{3}$ E-mail: fernandoaf@fvet.uba.ar; https://orcid.org/0000-0003-4425-9973
}

Recibido: 16 de julio de 2018

Aceptado para publicación: 20 de febrero de 2021

Publicado: 23 de junio de 2021

CLos autores. Este artículo es publicado por la Rev Inv Vet Perú de la Facultad de Medicina Veterinaria, Universidad Nacional Mayor de San Marcos. Este es un artículo de acceso abierto, distribuido bajo los términos de la licencia Creative Commons Atribución 4.0 Internacional (CC BY 4.0) [https:// creativecommons.org/licenses/by/4.0/deed.es] que permite el uso, distribución y reproducción en cualquier medio, siempre que la obra original sea debidamente citada de su fuente original 
Eucoleus boehmi (syn. Capillaria boehmi) is a trichurid nematode parasite of the upper respiratory tract in canids with a generally asymptomatic course. It is presented the clinical case of a 9-year-old mestizo male canine from the province of Buenos Aires, Argentina, who had previously been treated for eucoleosis, presenting cough, sneezing, reverse sneezing, abundant bilateral serous nasal discharge. The analysis of faecal samples was performed by the Bembrook and McMaster techniques, revealing the presence of 10900 epg of E. boehmi. Treatment and monitoring of the patient was performed until complete remission of clinical signs and the absence of eggs in the stool.

Key words: Eucoleus boehmi, dogs, EPG, eucoleosis

\section{INTRODUCCIÓN}

Eucoleus boehmi (sin. Capillaria boehmi) es un parásito nematode trichurideo del tracto respiratorio superior descrito por primera vez en zorros de la región de Moravia y Austria (Alho et al., 2016). Los vermes adultos se localizan en la mucosa de los cornetes nasales, los senos frontales y paranasales de los caninos domésticos, zorros y lobos (Campbell et al., 1991; Di Cesare et al., 2012; Veronesi et al., 2014). Su ciclo biológico y forma de transmisión no se conocen en detalle; sin embargo, se propone que los animales se infectan al ingerir los huevos larvados del parásito o por la ingesta de lombrices de tierra que podrían actuar como hospedadores intermediarios o paraténicos (Traversa et al., 2010; Veronesi et al., 2013; Di Cesare et al., 2015).

La eucoleosis por E. aerophilus es una enfermedad potencialmente zoonótica llamada capilariasis pulmonar en las personas (Laloševiæ et al., 2008). Si bien existen escasos registros a nivel mundial, la misma ha sido documentada en Rusia, Ucrania, Marruecos, Serbia, Irán y Francia (Coudert et al., 1972; Aftandelians et al., 1977; Cockshott y Middlemiss, 1979; Vilella et al., 1986). En cuanto a E. boehmi, su rol como agente zoonótico es incierto.
La infección en los caninos puede cursar de manera asintomática; sin embargo, en casos con altas cargas parasitarias, los animales pueden evidenciar signos clínicos como tos, estornudo, estornudo inverso, epistaxis, arcadas, descarga nasal e hipo o anosmia (Conboy et al., 2009; Traversa et al., 2010; Veronesi et al., 2013, 2014; Di Cesare et al., 2015;). Clark et al. (2013) registraron la presencia de convulsiones generalizadas en un canino debido a la migración errática de los vermes en el cerebro.

Los huevos de los parásitos de la familia Trichuridae se caracterizan por tener forma similar a un barril con tapones polares en sus extremos. La similitud presente entre los miembros de esta familia conlleva a dificultades en su diagnóstico. Los huevos de $E$. boehmi pueden medir 54-60 x 30-35 $\mu \mathrm{m}$, contienen un embrión multicelular, cámara de aire, color claro a amarillento y muestran una delicada superficie externa con hoyos (Campbell et al., 1991; Schoning et al., 1993; Di Cesare et al., 2012). En Argentina, Lavallén et al. (2018) realizaron la primera descripción molecular de E. boehmi en materia fecal de caninos naturalmente infectados de la Ciudad de Mar del Plata.

Debido a la falta de información respecto al seguimiento del tratamiento antihelmíntico en animales con recuentos al- 
tos de huevos por gramo de heces (hpg), el objetivo del presente trabajo fue describir un caso clínico de eucoleosis por E. boehmi en un canino con recuentos elevados de hpg.

\section{Descripción del Caso}

Un canino macho mestizo de 9 años (animal 1), entero, de $20 \mathrm{~kg}$ de peso, de la localidad de La Matanza (Villa Luzuriaga), Provincia de Buenos Aires, fue derivado por un profesional veterinario a la Cátedra de Parasitología y Enfermedades Parasitarias de la Universidad de Buenos Aires (UBA) para corroborar el diagnóstico y efectuar el manejo terapéutico de una reinfección por $E$. boehmi. El animal convivía con otro canino de 5 años (animal 2), en una casa con jardín con césped natural cercado de $20 \mathrm{~m}^{2}$, al que eventualmente accedían felinos.

Un año antes, el propietario había concurrido a la consulta con un profesional veterinario debido a que el animal presentaba tos y estornudos de manera permanente. El profesional realizó el examen clínico, sin encontrar otra particularidad más allá de los signos mencionados con anterioridad. Por ello, indicó realizar un análisis de sangre, un hisopado de cavidad nasal y una rinoscopía. El examen de sangre arrojó la presencia de eosinofilia (recuento relativo: 14\%, recuento absoluto: 1225 células $/ \mathrm{mm}^{3}$ ), en tanto que el cultivo bacteriológico y micológico de la muestra de la cavidad nasal fue negativo. En cuanto a la rinoscopía, se informó la presencia de mucosa congestiva y engrosada, con el hallazgo de vermes en los cornetes nasales derecho e izquierdo, emitiendo como diagnóstico «rinitis parasitaria». A partir de la observación de las características morfológicas de los vermes adultos extraídos de la cavidad nasal, utilizando un microscopio óptico, se identificó a E. boehmi (Supperer, 1953). Ante estos resultados, se prescribieron tres aplicaciones cada dos semanas de ivermectina $200 \mu \mathrm{g} / \mathrm{kg}$ por vía subcutánea.
Con el tratamiento aplicado, el paciente logró una mejoría de su estado general; sin embargo, los signos clínicos reaparecieron al cabo de tres meses, por lo cual el propietario consultó a otro profesional, quien decidió derivar la consulta al servicio de Parasitología y Enfermedades Parasitarias (UBA).

Al examen clínico, el animal presentaba tos, estornudos, estornudos inversos y abundante secreción nasal serosa bilateral. A la auscultación, los sonidos cardíacos y pulmonares no mostraron particularidades. $\mathrm{La}$ temperatura rectal fue de $38.4{ }^{\circ} \mathrm{C}$. No se observaron linfonódulos agrandados ni otra anomalía al examen particular.

Se indicó la recolección de materia fecal de cada can durante tres días consecutivos de los dos canes (si bien el animal 2 no presentaba signos clínicos, se realizó el muestreo para evaluar la existencia de una infección asintomática). Este procedimiento se llevó a cabo en las semanas $0,4,6,8,10$, $12,14,16,20,24,28$ y 32.

Cuadro 1. Valor medio del recuento de huevos por gramo (HPG) de E. boehmi presentes en heces de dos canes con eucoleosis por E. boehmi (Buenos Aires, Argentina)

\begin{tabular}{ccc}
\hline Semana & \multicolumn{2}{c}{ Valor medio de HPG } \\
\cline { 2 - 3 } & Animal 1 & Animal 2 \\
\hline 0 & 10,900 & 200 \\
4 & 7,800 & 0 \\
6 & 2,800 & 0 \\
8 & 2,166 & 0 \\
10 & 3,666 & 0 \\
12 & 1,433 & 0 \\
14 & 167 & 0 \\
16 & 0 & 0 \\
\hline El valor de & hpg en muestras de heces \\
colectadas cada cuatro semanas desde la \\
semana 16 a la 32 fue de 0 en los dos canes
\end{tabular}



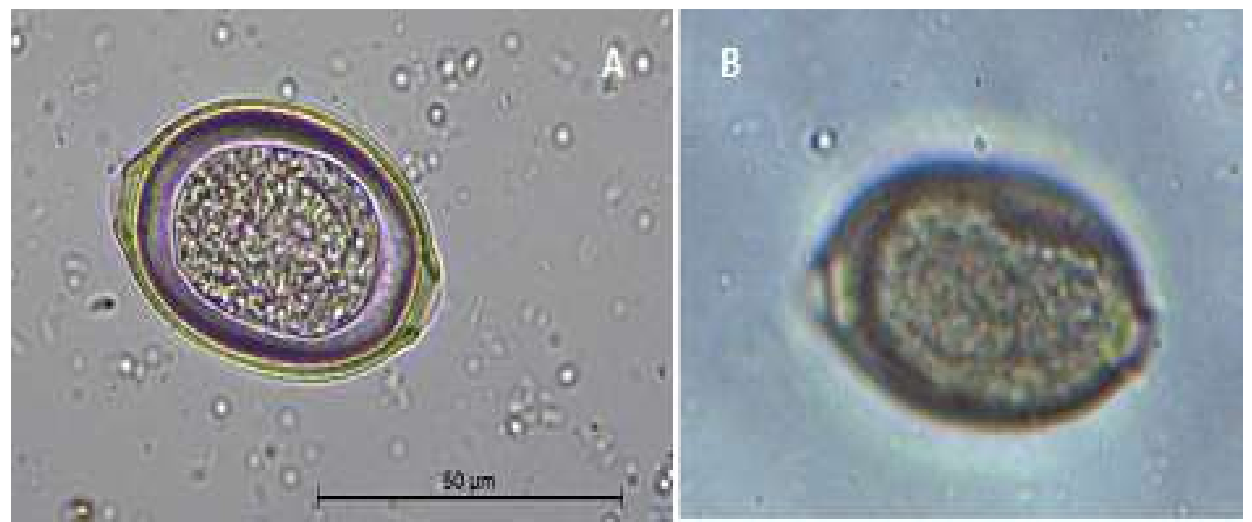

Figura 1. A. Huevo de Eucoleus boehmi, en forma de barril, de aproximadamente 50-60 $\mu \mathrm{m}$ de largo y 30-35 $\mu \mathrm{m}$ de ancho. Se observa tapones polares asimétricos, cámara de aire (40X). B. Membrana externa del huevo de E. boehmi que presenta pequeños hoyos brindando un aspecto poroso (40X)

Las muestras fueron procesadas a través de las técnicas de Benbrook y McMaster (Dolcetti et al., 1947; Permin y Hansen, 1998). En ambos animales se encontraron huevos en forma de barril, con cámara de aire evidente, tapones polares asimétricos, $\mathrm{y}$ pequeños hoyos en la superficie de la membrana externa brindando un aspecto poroso. Se tomaron microfotografías a través de la cámara ICC-50HD incorporada al microscopio LEICA ${ }^{\circledR}$ DM-500. Para efectuar las mediciones, se utilizó el módulo de medición interactiva del software LAS V4.5. Se obtuvieron valores promedio de $52.2 \mu \mathrm{m}$ de largo y $30.5 \mu \mathrm{m}$ de ancho (Figura 1), compatibles con E. boehmi (Di Cesare et al., 2012). Se encontró un valor de $10900 \mathrm{hpg}$ de $E$. boehmi para el animal 1 y de $200 \mathrm{hpg}$ para el animal 2 (Cuadro 1).

Se inició el tratamiento antihelmíntico con imidacloprid $10 \%$ y moxidectina $2.5 \%$ spot-on tal y como señalan Veronesi et al. (2014, 2017). Asimismo, dada la severidad de los signos clínicos del animal 1 , se instauró un tratamiento con prednisolona $1 \mathrm{mg} / \mathrm{kg}$ cada $12 \mathrm{~h}$ durante 10 días, para continuar con un esquema de días alternos.

Al mes, el animal 2 no presentaba huevos del parásito en las heces, mientras que el animal 1 tuvo $7800 \mathrm{hpg}$. Ante esto, se continuó con el tratamiento modificando el intervalo de aplicación cada dos semanas, observándose una reducción de los valores de hpg hasta la semana 10 en que se presentó un aumento de la carga parasitaria (Cuadro 1) y reaparición de signos clínicos (estornudos). Se reforzaron las indicaciones al propietario sobre la aplicación del producto spot-on (separar a los perros durante la administración, evitar que se frote o lama, permanecer junto al animal hasta verificar la absorción del producto). Estas medidas permitieron una reducción de la carga parasitaria hasta su negativización en la semana 16. A partir de allí, se espaciaron los tratamientos cada cuatro semanas, hasta observar cuatro resultados negativos (Cuadro 1). 
Discusión

El diagnóstico de muchas de las helmintiasis gastrointestinales y respiratorias de los caninos depende, entre otras cosas, de la correcta identificación de huevos o larvas de los parásitos en materia fecal. El reconocimiento inapropiado puede conducir a un diagnóstico erróneo y, en consecuencia, a un tratamiento incorrecto.

Este trabajo describe el primer caso clínico en el país de un canino naturalmente infectado por E. boehmi en el que se realizó la cuantificación de huevos en materia fecal, el tratamiento y el seguimiento durante un periodo de 32 semanas. Esto último resulta fundamental en el manejo de la parasitosis, ya que los animales enfermos o portadores constituyen una fuente importante de contaminación, que sumado a la elevada resistencia ambiental que poseen los huevos de Eucoleus spp, pueden contribuir a la reinfección de los animales luego de aplicado el tratamiento (Veronesi et al., 2013).

Considerando la transmisión fecal-oral y la resistencia de los huevos, las medidas sanitarias como la remoción de las heces del ambiente, la prevención de las prácticas de geofagia y coprofagia, resultan procedimientos cruciales para controlar la enfermedad y evitar la reinfección de los animales (Baan et al., 2011). Los caninos pueden contraer infecciones por helmintos del orden Trichocephalida, entre los que se encuentra Trichuris vulpis, Eucoleus boehmi, Eucoleus aerophilus. Si bien los estadios adultos se localizan en intestino grueso, cavidad nasal y pulmón, respectivamente, los huevos se liberan junto con la materia fecal y poseen características morfológicas similares, lo que configura un importante desafío diagnóstico, tanto en el escenario epidemiológico como clínico (Di Cesare et al., 2012).
Si bien existen investigaciones con respecto a protocolos de tratamiento para $E$. boehmi, las mismas son escasas. La administración de una única dosis por vía oral de ivermectina de $200 \mu \mathrm{g} / \mathrm{kg}$ o de milbemicina oxima de $2 \mathrm{mg} / \mathrm{kg}$, así como la administración de fenbendazol por vía oral a dosis de 50 $\mathrm{mg} / \mathrm{kg}$ cada $24 \mathrm{~h}$ durante 14 días se han reportado como efectivas para el tratamiento (Alho et al., 2016). Por otro lado, Veronesi et al. (2014) demostraron la eficacia contra $E$. boehmi en perros de una formulación spoton de $10 \%$ imidacloprid $/ 2.5 \%$ moxidectina.

La inclusión de Eucoleus spp en el diagnóstico diferencial de las enfermedades cardiorrespiratorias de los caninos es importante. El análisis coproparasitológico de rutina por técnicas de flotación resulta suficiente para evidenciar la presencia de huevos de trichurideos. La correcta identificación y medición de las características morfológicas permite confirmar el diagnóstico de eucoleosis ante un caso sospechoso o detectar la presencia de una infección asintomática.

\section{Agradecimientos}

Al Dr. Alberto Carugati por la derivación del caso a la Cátedra de Parasitología y Enfermedades Parasitarias de la Facultad de Ciencias Veterinarias de la Universidad de Buenos Aires.

\section{Limeratura Citada}

1. Aftandelians R, Raafat F, Taffazoli M, Beaver PC, 1977. Pulmonary capillariasis in a child in Iran. Am J Trop Med Hyg 26: 64-71. doi: 10.4269/ajtmh.1977.26.64

2. Alho AM, Mouro S, Pissarra H, Murta A, Lemos M, Gomes L, Lima C, et al. 2016. First report of Eucoleus boehmi infection in a dog from Portugal. Parasitol Res 115: 1721-1725. doi: 10.1007/s00436-016-4932-6 
3. Baan M, Kidder AC, Johnson SE, Sherding RG. 2011. Rhinoscopic diagnosis of Eucoleus boehmi infection in a dog. J Am Anim Hosp Assoc 47: 60-63. doi: 10.5326/JAAHA-MS-5707

4. Campbell BG, Little MD. 1991. Identification of the eggs of a nematode (Eucoleus boehmi) from the nasal mucosa of North American dogs. J Am Vet Med Assoc 198: 1520-1523.

5. Clark AC, López FR, Levine JM, Cooper JJ, Craig TM, Voges AK, Johnson MC, et al. 2013. Intracranial migration of Eucoleus (Capillaria) boehmi in a dog. J Small Anim Pract 54: 99-103. doi: 10.1111/j.1748-5827.2012.01303.x

6. Cockshott WP, Middlemiss JH. 1979. Clinical radiology in the tropics. Edinburgh: Churchill Livingstone Press. 232 p.

7. Conboy G. 2009. Helminth parasites of the canine and feline respiratory tract. Vet Clin NAm-Small 39: 1109-1126. doi: 10.1016/j.cvsm.2009.06.006

8. Coudert J, Despeignes J, Battesti R. 1972. A propos d'un cas de capillariose pulmonaire. Bull Soc Pathol Exot 65: 841-848.

9. Di Cesare A, Castagna G, Meloni S, Otranto D, Traversa D. 2012. Mixed trichuroid infestation in a dog from Italy. Parasite Vector 5: 128. doi: 10.1186/17563305-5-128

10. Di Cesare A, Veronesi F, di Regalbono $A F$, De Liberato C, Perrucci S, Iorio $R$, Morganti G, et al. 2015. PCR-based assay for the mitochondrial cox 1 specific amplification of Eucoleus böhmi. Vet Parasitol 211: 67-70. doi: 10.1016/ j.vetpar.2015.04.016

11. Dolcetti M. 1947. Modificación al método de Benbrook mediante la centrifugación doble con solución de azúcar para el examen microscópico de las materias fecales de los animales domésticos. Rev CE Med 16: 29-35.

12. Laloševice D, Laloševice V, Klem I, Stanojev-Jovanovice D, Pozio E. 2008. Pulmonary capilariasis miming bronchial carcinoma. Am J Trop Med Hyg 78: 14-16.
13. Lavallén CM, Petrigh RS, Fugassa MH, Denegri GM, Dopchiz MC. 2018. First morphological and molecular analysis of Eucoleus boehmi like eggs in dogs from Argentina. Parasitol Res 117: 2351-2357. doi: 10.1007/s00436018-5918-3

14. Permin A, Hansen JW. 1998: The epidemiology, diagnosis and control of poultry parasites. Rome: FAO. $160 \mathrm{p}$.

15. Schoning P, Dryden MW, Gabbert NH. 1993. Identification of a nasal nematode (Eucoleus boehmi) in greyhounds. Vet Res Commun 17: 277-281. doi: 10.1007/ BF01839218

16. Supperer R. 1953. Capillaria böhmi spec. nov., eine neue haarwurmart aus den stirnhöhlen des fuchses. Zeitschrift für Parasitenkunde 16: 51-55.

17. Traversa D, Di Cesare A, Conboy G 2010. Canine and feline cardiopulmonary parasitic nematodes in Europe: emerging and underestimated. Parasite Vector 3: 62. doi: 10.1186/1756-3305-3-62

18. Veronesi F, Di Cesare A, Braun G, Günther L, Morganti G, Rueca F, Petry G, et al. 2017. Evaluation of the clinical efficacy and safety of a spot-on combination of imidacloprid 10\%/ Moxidectin 2.5\% (Advocate ${ }^{\circledR}$, Advantage ${ }^{\circledR}$ Multi) in comparison to an untreated control group in the treatment of Capillaria boehmi in naturally infected dogs. Parasitol Res 116(Suppl 1): 65. doi: 10.1007/s00436-017-5492-0

19. Veronesi F, Lepri E, Morganti G, Di Palma S, Mechelli L, Moretti A, Traversa D. 2013. Nasal eucoleosis in a symptomatic dog from Italy. Vet Parasitol 195: 187-191. doi: 10.1016/ j.vetpar.2013.01.022

20. Veronesi F, Morganti G, di Cesare A, Lepri E, Cassini R, Zanet S, Deni D, et al. 2014. Eucoleus boehmi infection in red fox (Vulpes vulpes) from Italy. Vet Parasitol 206: 232-239. doi: 10.1016/ j.vetpar.2014.10.001

21. Vilella JM, Desmaret MC, Rouault E. 1986. Capillariose à Capillaria aerophila chez un adulte? Med Mal Infect 1:35-36. 